Gastroenterologia 1965;104(Suppl. 1):5

\title{
Pathogenese der akuten und chronischen Pankreatitis
} W. Doerr

Aus dem Pathologischen Institut der Universität Heidelberg (Dir.: Prof. Dr. W. Doerr)

Hier finden sich kurze Zusammenfassungen dieser Referate und Vorträge. E. Es wird eine umfassende Analyse des pathogenetischen Geschehens bei der akuten und chronischen Pankreatitis aus der Sicht des Pathologen gegeben. Nach einer Übersicht über die normale Histophysiologie des Pankreas wird eine Aufgliederung der entzündlichen Pankreaserkrankungen in 3 Formenkreise vorgenommen: 1. metabolische Läsionen 2. begleitende Pankreasentzündungen

3. organeigentüm-liche Prozesse. Die einzelnen Formen werden ausführlich mit Beispielen belegt

wo-bei auf verschiedenste pathogenetische Vorstellungen über die Pankreatitis kritisch eingegangen wird. In einem weiteren Abschnitt werden die Voraussetzungen und Möglichkeiten der experimentellen Pankreatitisforschung dargelegt. Arbeiten aus dem Arbeitskreis des Verfassers werden in diesem Zusammenhang diskutiert. Das umfangreiche Referat schließt mit einem Ausblick auf aktuelle Fragen der Pankreatitisforschung. Das Literaturverzeichnis umfaßt 304 Titel. Die Referate und Vorträge die bei der gemeinsamen Sitzung mit der Deutschen Gesellschaft für innere Medizin am Vormittag des 9. 